\title{
A well-monitored, X-ray selected, tidal disruption event
}

\author{
R.D. Saxton ${ }^{1, a}$, A.M. Read ${ }^{2}$, S. Komossa ${ }^{3}$, and P. Esquej ${ }^{4}$ \\ ${ }^{1}$ XMM SOC, ESAC, Apartado 78, 28691 Villanueva de la Cañada, Madrid, Spain \\ 2 Dept. of Physics and Astronomy, University of Leicester, Leicester LE1 7RH, UK \\ ${ }^{3}$ Max-Planck-Institut für Radioastronomie, Auf dem Hügel 69, 53121 Bonn, Germany \\ ${ }^{4}$ Centro de Astrobiología (CSIC-INTA), 28850 Torrejón de Ardoz, Madrid, Spain
}

\begin{abstract}
We report on a candidate tidal disruption event detected in the XMM-Newton slew survey from the nucleus of SDSS J120136.02+300305.5 $(z=0.146$; hereafter SDSS J1201+30). The source, monitored by Swift and XMM-Newton, was highly variable on timescales of a week, reaching a peak X-ray luminosity of $3 \times 10^{44} \mathrm{ergs} / \mathrm{s}$. The light curve is reminiscent of the variations seen in SWIFT J1644+57, although in this case the absence of radio flux rules out a jet mechanism for the emission. The X-ray spectrum is steep, (spectral index $=3-5$ ) and softens with diminishing flux. It is inconsistent with a single or multi-temperature black-body model but may be fit with Bremsstrahlung or comptonised thermal emission.
\end{abstract}

\section{INTRODUCTION}

A tidal disruption event (TDE) occurs when a stellar object is gravitationally destroyed and subsequently accreted by a super-massive black hole (SMBH). It represents the onset of a brief phase of AGN activity in an otherwise dormant galactic nucleus and is characterised by a sudden dramatic rise in luminosity, and a decline to quiescence on a timescale of months to years.

It is important to catch a TDE around the peak of its activity where the photon statistics are good and where differences occur which can help to distinguish between models. To this end we have initiated a program to monitor sources found in XMM-Newton slews [10] and compare their flux quickly with that seen in ROSAT. Extragalactic sources, which show a strong increase in flux, and are not known classical AGN, then become candidates for a follow-up program. Such sources are very rare. We report here on the galaxy SDSS J120136.02+300305.5, observed during an XMM-Newton slew with a flux 56 times greater than the upper limit from the ROSAT all-sky survey (RASS; [18]).

\section{X-RAY FLARE IDENTIFICATION}

During a slew, performed on June $10^{\text {th }}$ 2010, XMMNewton detected a source, XMMSL1 J120135.7+ 300306, with an EPIC-pn, medium filter, 0.2-2 keV count rate of $2.1 \pm 0.5$ count s$^{-1}$. For a typical theoretical tidal disruption spectrum of a black body of temperature $70 \mathrm{eV}$ and the Galactic column for this sky position of $1.4 \times 10^{20} \mathrm{~cm}^{-2}$ [4], this corresponds to an unabsorbed flux of $\mathrm{F}_{0.2-2.0}=$ $3.1 \times 10^{-12} \mathrm{ergs} \mathrm{s}^{-1} \mathrm{~cm}^{-2}$. We calculate a 2-sigma upper limit from the RASS at this position of 0.03 count s ${ }^{-1}$ (see [2] for a description of the upper limit calculation), corresponding to $\mathrm{F}_{0.2-2.0} \leq 5.4 \times 10^{-14} \mathrm{ergs} \mathrm{s}^{-1} \mathrm{~cm}^{-2}$, using the same spectral model; a factor 56 below the XMMNewton slew value.

a e-mail: richard.saxton@sciops.esa.int

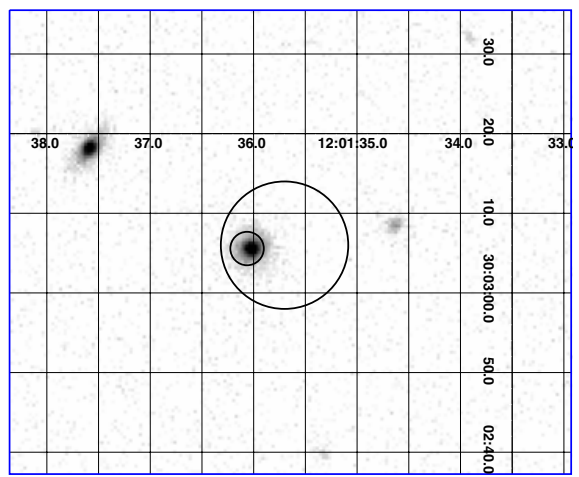

Figure 1. An r-filter image of the galaxy from the SDSS survey, (limiting magnitude $r \sim 23$ ), taken on 2004-1213, shown with the XMM-Newton slew error circle (8 arcsecond radius) and UVOT-enhanced Swift error circle (2.1 arcsecond radius; see text) centred on the detections.

\section{X-RAY FOLLOW-UP OBSERVATIONS}

An X-ray monitoring program was initiated with Swift to follow the evolution of the source flux and spectrum. Snapshot 3ks observations were made once a week with the Swift-XRT and the UV optical telescope (UVOT). An accurate position for the source in the Swift-XRT field can be determined by matching the UVOT field of view with the USNO-B1 catalogue and showed that the source is coincident with the galactic nucleus (see Fig. 1). In parallel, 30ks XMM-Newton pointed observations were triggered on 2010-06-22, 2010-11-23 and 2010-12-23. First results from these observations have been published in [12]. Further analyses, presented here, have been made with the XMM-Newton Science Analysis System (SAS v12; [3]). Light curves were extracted from the observations and searched for periods of high background flaring. These periods were excluded from further data analysis and the total amount of remaining exposure time is listed in Table 1, together with details of the Swift observations. 
Table 1. X-ray observation log of SDSS J1201+30.

\begin{tabular}{llll}
\hline Mission $^{a}$ & Date & $\begin{array}{l}\text { Exp time }^{b} \\
(\mathrm{~s})\end{array}$ & $\begin{array}{l}\text { Flux }^{c} \\
\left(10^{-12} \mathrm{ergs} \mathrm{s}^{-1} \mathrm{~cm}^{-2}\right)\end{array}$ \\
\hline ROSAT & 1990 & 500 & $<0.054$ \\
XMM slew & $2010-06-10$ & 8.9 & $4.7_{-2.0}^{+3.4}$ \\
SWIFT & $2010-06-20$ & 2996 & $0.56 \pm 0.26$ \\
XMM pointed & $2010-06-22$ & 18865 & $0.81 \pm 0.07$ \\
SWIFT & $2010-06-30$ & 3252 & $1.4 \pm 0.4$ \\
SWIFT & $2010-07-07$ & 3580 & $<0.030$ \\
SWIFT & $2010-07-14$ & 2780 & $<0.039$ \\
SWIFT & $2010-07-21$ & 3065 & $<0.035$ \\
SWIFT & $2010-07-28$ & 505 & $<0.21$ \\
SWIFT & $2010-10-24$ & 3189 & $0.13 \pm 0.05$ \\
XMM pointed & $2010-11-23$ & 10697 & $0.19 \pm 0.03$ \\
XMM pointed & $2010-12-23$ & 11303 & $0.04 \pm 0.02$ \\
SWIFT & $2011-03-25$ & 2285 & $<0.046$ \\
SWIFT & $2011-04-01$ & 2912 & $<0.035$ \\
SWIFT & $2011-04-11$ & 2688 & $<0.039$ \\
\hline
\end{tabular}

${ }^{a}$ XMM-Newton, EPIC-pn camera: slew observation performed in full frame mode with the Medium filter; pointed observations performed in full frame mode with the thin1 filter. Swift-XRT in pc mode.

${ }^{b}$ Useful exposure time after removing times of high background flares.

$c$ Unabsorbed flux, $F_{0.2-2 \mathrm{keV}}$ calculated with the best fit Bremsstrahlung model (or $k T=300 \mathrm{eV}$ for the upper limits; see Section 4.3) and Galactic absorption of $1.4 \times 10^{20} \mathrm{~cm}^{-2}$.

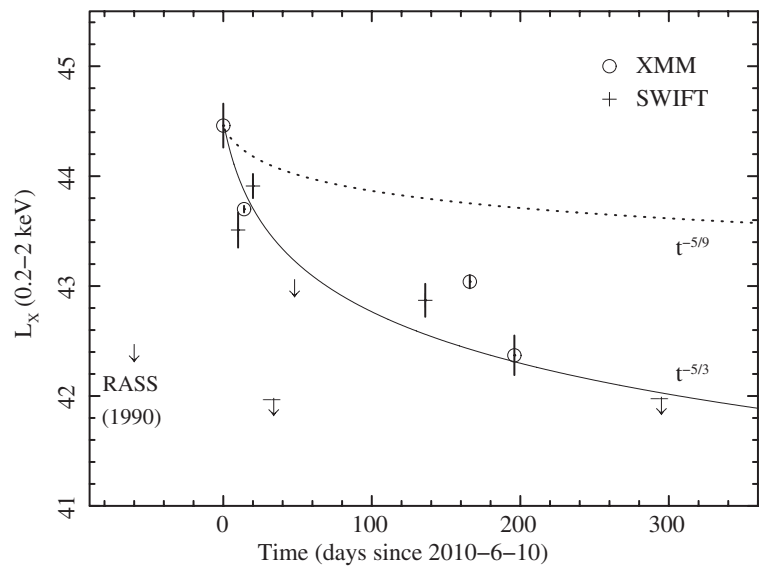

Figure 2. The $0.2-2 \mathrm{keV}$ long-term $\mathrm{X}$-ray light curve of SDSS J1201+30. XMM-Newton slew and pointed observations: circle, Swift-XRT: cross. All upper limits are from Swift except the RASS point. The first Swift upper limit point is calculated by combining data from the 2010-07-07, 2010-07-14 and 201007-21 observations, while the last upper limit is obtained from a combination of the 2011-03-25, 2011-04-01 and 2011-04-11 observations. The lines represent $\mathrm{t}^{-5 / 3}$ (solid) and $\mathrm{t}^{-5 / 9}$ (dotted) declines.

\subsection{X-ray light curve}

In figure 2 we show the long-term $\mathrm{X}$-ray light curve for SDSS $J 1201+30$. The source is highly variable, with a factor 50 drop between 2010-06-30 and 2010-07-07 and a factor 80 drop in flux between 2010-06-30 and 2010-0714 if we calculate an upper limit by combining the Swift observations of 2010-07-07, 2010-07-14 and 2010-07-21.

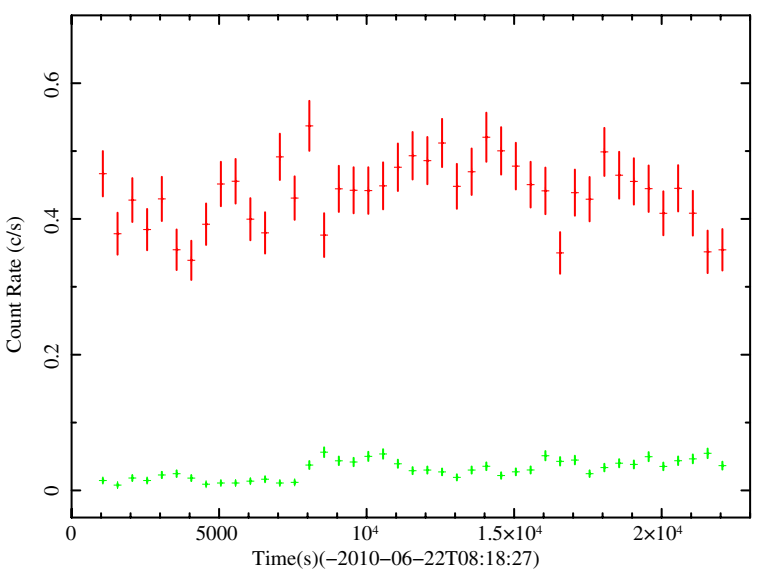

Figure 3. The background-subtracted, exposure corrected, EPICpn (top) $0.16-1.0 \mathrm{keV}$ light curve from the first XMM-Newton pointed observation of 2010-06-22, together with the exposure corrected, EPIC-pn background (bottom), which reaches a maximum of 0.055 count $\mathrm{s}^{-1}$.

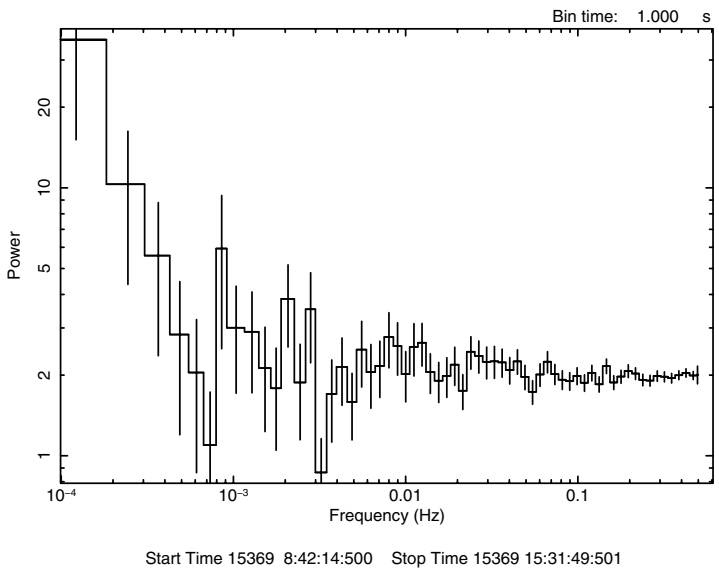

Figure 4. Power spectrum for the XMM-Newton observation of 2010-06-22, in the $0.2-2 \mathrm{keV}$ energy range.

We have superimposed two fiducial decay curves onto the $\mathrm{X}$-ray light curve: a canonical $\mathrm{t}^{-5 / 3}$ evolution related to the rate of return of tidal debris and a $\mathrm{t}^{-5 / 9}$ decline which is that predicted for EUV emission from an expanding wind ([15]). While the strong variations make it impossible to fit a smooth curve through the data points, the overall decay appears to be steeper than the $\mathrm{t}^{-5 / 9}$ law but not inconsistent with a $\mathrm{t}^{-5 / 3}$ decline.

The source is variable on timescales of a few thousand seconds (Fig. 3). This rather slow variability implies a relatively high black hole mass and following the prescription of [7] we calculate $M_{B H}=2.5 \times 10^{7} M_{\odot}$ from the excess variance with a systematic error of 0.4 dex. It should be pointed out that the correlation of excess variance with $M_{B H}$ in [7] has been calculated using persistent AGN and the disk conditions, leading to the $\mathrm{X}$-ray variability, are not necessarily the same in TDE. From the relationship of black hole mass to bulge K-band luminosity ([6]) and using a bulge to total-light luminosity ratio for SDSS J1201+30 of $85 \%$ ([13]), we find $M_{B H}=$ $1.8 \times 10^{7} M_{\odot}$, in good agreement with the estimate based on variability. 
Table 2. Spectral fits to the 2010-06-22 XMM-Newton, EPIC-pn, observation of SDSS J1201+30.

\begin{tabular}{|c|c|c|c|c|c|}
\hline \multirow[t]{2}{*}{ Model $^{a}$} & \multicolumn{2}{|c|}{ emission } & \multicolumn{2}{|c|}{ zxipcf } & \multirow[t]{2}{*}{$\mathrm{C} / \mathrm{dof}$} \\
\hline & $\mathrm{kT}(\mathrm{eV})^{b}$ & $\Gamma$ & $\mathrm{NH}^{c}$ & $\log (\mathrm{xi})^{d}$ & \\
\hline Diskbb & $70^{*}$ & - & - & - & $5510 / 335$ \\
\hline Pow+BB & $70^{*}$ & $3.33 \pm 0.05$ & - & - & $465 / 333$ \\
\hline Brem & $373 \pm 11$ & - & - & - & $372 / 334$ \\
\hline Brem & $366 \pm 13$ & - & $7_{-6}^{+5}$ & $0.4_{-0.2}^{+0.5}$ & $353 / 332$ \\
\hline Comptt $^{e}$ & $75 \pm 5$ & - & - & - & $375 / 333$ \\
\hline Compbb $^{e}$ & $69 \pm 6$ & - & - & - & $363 / 333$ \\
\hline
\end{tabular}

a The xspec emission models consisting of multi-coloured disk black-body (diskbb), power-law plus black-body, Bremsstrahlung, comptt and compbb. All models were fit with the Galactic absorption value of $1.4 \times 10^{20} \mathrm{~cm}^{-2}$ ([4]). Only in the case of Bremsstrahlung did the addition of an ionised absorber model (zxipcf) improve the fit.

${ }^{b}$ Temperature of the thermal component in the source rest frame. ${ }^{c}$ Column density in the ionized absorber component $\left(10^{20} \mathrm{~cm}^{-2}\right)$.

${ }^{d} \log (\mathrm{xi})$ where $\mathrm{xi}=L / \mathrm{n} r^{2}$.

${ }^{e}$ The electron temperature of the hot comptonising plasma was fixed to $2 \mathrm{keV}$.

* Temperature frozen to $70 \mathrm{eV}$. Freeing the parameter did not improve the fit.

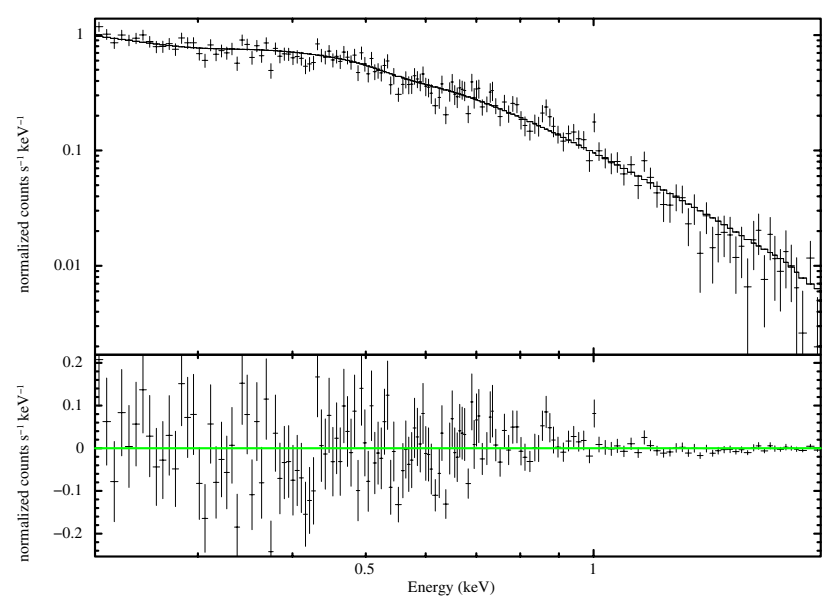

Figure 5. A spectral fit to the XMM-Newton, EPIC-pn, observation of 2010-06-22, using a comptonised black-body model.

Quasi-periodic variability (QPO) has been claimed for SWIFT J1644+57 by [8]. We have searched for similar variability in SDSS J1201+30 without finding a significant peak (Fig. 4).

The strong, weekly variability is similar to that seen in SWIFT J1644+57. In the latter case the explanations have focussed on jet instabilities for the most part (e.g. [9]) although the disruption of a compact object has also been proposed ([5]). A deep eVLA radio observation of SDSS J1201+30 excluded the presence of a jet in this source. One possible common mechanism for the strong variability is Lense-Thirring precession of the accretion structure ([14]). For SDSS J1201+30 the precession period would need to be $>50$ days, which would imply a rapidly rotating Black hole for $M_{B H} \sim 10^{7} M_{\odot}$. Clumpy accretion is a further possibility. The time needed to consume a debris disk is given by $T=180 M_{7} \dot{m}_{0.1}^{-2} r_{10}^{7 / 2}$ days (where $r_{10}$ is the radius of the inner disk in units of $10 R_{s}$; [17]). For $M_{B H}=2 \times 10^{7} M_{\odot}, L_{b o l} \approx 3 \times 10^{44} \mathrm{ergs} / \mathrm{s}$ (assuming an X-ray to bolometric ratio of 3 ) then $\dot{m} \approx 0.1$ and for the disk to be consumed in 7 days (2010-06-30 to 2011-07-07) the disk inner radius would need to be at $3 R_{s}$.

\subsection{X-ray spectrum}

In [12] we showed that the combined EPIC-pn, and MOS spectra for the three XMM-Newton observations are not well modelled by multi-coloured black-body emission. They were fit well by Bremsstrahlung radiation whose temperature declined from $390 \pm 10$ to $290 \pm 20$ to $180_{-30}^{+60} \mathrm{eV}$ as the X-ray luminosity dropped from $4.7 \times 10^{43}$ to $1.1 \times 10^{43}$ to $2.3 \times 10^{42} \mathrm{ergs} / \mathrm{s}$. This is consistent with the $L \propto T^{4}$ relationship of thermally emitting material. A solar-abundance plasma, emitting opticallythin (Bremsstrahlung) radiation with $k T=390 \mathrm{eV}$ would emit strong line radiation which is not evident in the spectrum. While the light elements are unconstrained, Nitrogen is limited to $10 \%$ and Oxygen to just $2 \%$ of solar abundance.

The inferred density of material in the emitting inner region is high enough that it should be opticallythick, giving a natural explanation for the absence of emission line features. As black-body models do not fit the spectrum, comptonisation by a low-energy electron plasma, as proposed for the soft excess seen in most AGN (e.g. [1]), may be involved. Here we concentrate on the first XMM-Newton observation and restrict the analysis to the EPIC-pn data only. The results of spectral fits to these data are given in Table 2. The comptonisation models, compbb and comptt give a reasonable fit (Fig. 5) but a Bremsstrahlung with a small ionized absorber $\left(N_{H}=\right.$ $7_{-6}^{+5} \times 10^{20} \mathrm{~cm}^{-2}$ ) is a little better.

\section{SUMMARY}

The X-ray spectrum of SDSS J1201+30 is similar to that of a small number of low black-hole-mass AGN which appear to have a 'soft excess' but no hard X-ray power law (e.g. SDSS J1231+1106; [16] and GSN 069; [11], Miniutti et al. in prep.). While the X-ray emission is likely to be produced by a form of comptonisation of disk photons the exact form needs to be identified before physical parameters can be extracted. Looking to the future, the statistics and spectral resolution of the first XMM-Newton pointed observation do appear to be sufficient for accurate spectral fitting. A longer observation would not necessarily help the spectral determination while a brighter (closer) source, allowing a grating spectrum to be made, would aid the determination of absorption components but probably not the underlying emission. However, a closer source which allowed the optical/UV emission from the TDE to be extracted simultaneously with the X-rays might enable self-consistent disk models to be fit (e.g. [1]). A longer XMM-Newton observation, taken near the peak, would improve the accuracy of the temporal power spectrum increasing the possibility of finding features such as QPOs. 


\section{References}

[1] Done, C., Davis, S., Jin, C., Blaes, O., Ward, M., MNRAS 420, (2012) 1848

[2] Esquej, P., Saxton, R., Freyberg, M. et al. A\&A 462, (2007) 49

[3] Gabriel, C. et al. :, In ASP Conf. Ser., Vol. 314, ADASS Xiii ed. Oschenbein, F., Allen, M. \& Egret, D. (2003) 759.

[4] Kalberla, P., Burton, W., Hartmann, D. et al., A\&A 440, (2005) 775

[5] Krolik, J. \& Piran, T., ApJ 743, (2011) 134

[6] Marconi, A. \& Hunt, L. ApJ, 589 (2003) L21

[7] Ponti, G., Papadakis, I., Bianchi, S., et al. A\&A 542, (2012) 83

[8] Reis, R., Miller, J., Reynolds, M., et al. Sci 337, (2012) 949

[9] Saxton, C., Soria, R., Wu, K., Kuin, N., MNRAS 422 , (2012) 1625
[10] Saxton, R., Read, A., Esquej, P. et al. , A\&A 480, (2008) 611

[11] Saxton, R., Read, A., Esquej, P., Miniutti, G. Alvarez, E., In: "Narrow-Line Seyfert 1 Galaxies and Their Place in the Universe", Proceedings of Science, NLS1, (2011) 008

[12] Saxton, R., Read, A., Esquej, P., Komossa, S. et al. A\&A 541, (2012) 106

[13] Simard, L., Mendel, L., David, R. et al. ApJS 196, (2011) 11

[14] Stone, N. \& Loeb, A. PhRvL 108, (2012) 1302

[15] Strubbe, L.E. \& Quataert, E., MNRAS 400, (2009) 2070

[16] Terashima, Y., Kamizasa, N., Awaki, H., Kubota, A., Ueda, Y., ApJ 752, (2012) 154

[17] Wang, J.-M., Cheng, C. \& Li, Y.-R., ApJ 748 (2012) 147.

[18] Voges, W., Aschenbach, B., Boller, T. et al., A\&A 349, (1999) 389 